\title{
Expression analysis of the autosomal recessive primary microcephaly genes MCPH1 (microcephalin) and MCPH5 (ASPM, abnormal spindle-like, microcephaly associated) in human malignant gliomas
}

\author{
CARSTEN HAGEMANN $^{1}$, JELENA ANACKER ${ }^{2}$, STEFANIE GERNGRAS ${ }^{1}$, \\ SIGLINDE KÜHNEL ${ }^{1}$, HARUN M. SAID ${ }^{3}$, RAJNIKANT PATEL ${ }^{4}$, ULRIKE KÄMMERER ${ }^{2}$, \\ DIRK VORDERMARK ${ }^{3}$, KLAUS ROOSEN $^{1}$ and GILES HAMILTON VINCE ${ }^{1}$ \\ ${ }^{1}$ Department of Neurosurgery, Tumor Biology Laboratory, ${ }^{2}$ Departments of Obstetrics and Gynaecology, \\ ${ }^{3}$ Radiation Oncology, University of Würzburg, D-97080 Würzburg, Germany; ${ }^{4}$ Department of Biochemistry, \\ University of Leicester, Henry Wellcome Building, Leicester LE1 9HN, UK
}

Received January 24, 2008; Accepted April 4, 2008

DOI: $10.3892 /$ or_00000007

\begin{abstract}
Patients with autosomal recessive primary microcephaly have a small but architecturally normal brain containing a reduced number of neurons. Microcephalin and $A S P M$ are two of the genes causing this disease. Both are centrosomal proteins involved in cell cycle regulation. Whereas microcephalin is a component of the DNA damage response and a repressor of telomerase function, ASPM is required for the proper formation of a central mitotic spindle and ensures symmetric, proliferative divisions of neuroepithelial cells. Both proteins are also involved in the regulation of tumor growth. Microcephalin expression is reduced in breast cancer cell lines and human tumors of the ovary and prostate. Reduction in microcephalin mRNA expression correlates with increased chromosomal instability. ASPM mRNA is overexpressed in transformed human cell lines and tumors, and its increased expression is positively associated with proliferation of glioblastoma cells. Glioblastomas are the most prevalent malignant brain tumors in adults, characterized by increased invasiveness, an aggressive local growth pattern and short survival periods of patients. In this study, we analysed the expression of microcephalin mRNA and ASPM mRNA and protein in a panel of 15 glioblastomas and 15 astrocytoma WHO grade II by semiquantitative RT-PCR, Western blotting and immunohisto-
\end{abstract}

Correspondence to: Dr Carsten Hagemann, Department of Neurosurgery, Tumor Biology Laboratory, University of Würzburg, Josef-Schneider-Str. 11, D-97080 Würzburg, Germany

E-mail: hagemann_c@klinik.uni-wuerzburg.de

Key words: astrocytoma, abnormal spindle-like microcephaly associated (ASPM, MCPH5), autosomal recessive primary microcephaly, expression analysis, glioblastoma multiforme, glioma, microcephalin chemistry. Whereas microcephalin expression did not seem to be altered during glioma development, there was a clear increase in ASPM mRNA and protein expression that corresponded with the WHO grade of the tumor. Our findings are significant as the expression of ASPM may be used as a marker for glioma malignancy and represents a potential therapeutic target.

\section{Introduction}

Autosomal recessive primary microcephaly (MCPH) (MIM $251200)$ is a disorder of fetal brain growth leading to microcephaly at birth accompanied by nonprogressive mental retardation. Patients have a small but architecturally normal brain whose cerebral cortex exhibits the greatest size reduction. Aetiologically, this miniaturization most likely reflects a reduction in neural cell number caused by reduced neuron production during fetal life (1). At least six MCPH gene loci have been detected and four genes identified and analysed in detail (2). Two of the genes, microcephalin (MCPH1; also known as BRCT-repeat inhibitor of hTERT expression, BRIT1) and ASPM (abnormal spindle-like, microcephaly associated; $M C P H 5)$ are the subject of the present analysis.

The microcephalin gene was the first to be identified as a direct cause of MCPH in two consanguineous families of Pakistani origin by autozygosity mapping $(3,4)$. Originally, microcephalin was found to serve as a repressor of human telomerase function (5). However, recent studies have shown that it is an important component of the DNA damage response network. Microcephalin is a centrosomal protein (6) and prevents premature chromosome condensation (7) by negatively regulating condensin II (8). Microcephalin is also required for the correct function of the intra-S phase and the $\mathrm{G} 2 / \mathrm{M}$ checkpoints in response to ionizing radiation and DNA damage (9-12). It has been suggested that microcephalin may function in ataxia and telangiectasia and the Rad3-related (ATR) signalling pathway $(11,12)$. There is an inverse 
relationship between microcephalin expression and genomic instability (12). Its expression is significantly reduced in several breast cancer cell lines and human epithelial tumors of the ovary and prostate (12). This has led to the belief that microcephalin may act as a novel tumor suppressor $(9,12)$.

Whereas the microcephalin gene was the first identified cause of MCPH, homozygous mutations in the ASPM gene $(13,14)$ are the most common reason for MCPH development $(2,15)$. ASPM is specifically expressed at the primary sites of prenatal cerebral cortical neurogenesis in mice (16). It is a centrosomal protein during interphase and concentrates at the spindle poles between prophase and telophase of the cell cycle $(17,18)$. Abnormal spindles (Asp), the ortholog of ASPM in Drosophila, is essential for the organization and bundling of microtubules at the spindle poles and has been shown to be necessary for the proper formation of a central mitotic spindle during both mitosis and meiosis $(2,15)$. Mouse embryonic neuroepithelial (NE) cells, the primary stem and progenitor cells of the mammalian brain, show two distinct types of cell division. During brain development NE cells perform symmetric, proliferative divisions, thereby increasing the number of progenitor cells. Later, a cell fate decision occurs; hence the cleavage plane orientation is shifted, and asymmetric neurogenic divisions take place. ASPM plays a critical role at the spindle poles of NE cells in maintaining spindle position during mitosis to ensure symmetric cell divisons (19). ASPM mRNA is generally expressed in proliferating tissues, not only during embryonic development, but also in a multitude of adult human tissues, although at much lower concentrations than in fetal tissues $(17,20)$. Only in the adult human brain and in skeletal muscle is ASPM mRNA missing $(17,20)$. ASPM is highly overexpressed in transformed human cell lines, cancer cell lines, and several tumor types as determined by high-density oligonucleotide microarrays and RT-PCR $(17,20)$. Recently, Horvath et al performed a weighted genecoexpression network analysis on RNA from 120 glioblastoma (GBM, WHO grade IV) patient samples and identified ASPM as a key gene within a gene coexpression module (21). ASPM mRNA was overexpressed in GBM relative to normal brain or body tissues (21-23), and siRNAmediated depletion of ASPM in cultured cells resulted in G1phase cell cycle arrest (21).

GBMs are the most prevalent malignant brain tumors in adults. They may manifest de novo or progress from a lowgrade astrocytoma (LGA, WHO grade II) or anaplastic astrocytoma (WHO grade III) (24) and are characterized by a high degree of invasiveness and an aggressive local growth pattern. The overall median survival time for GBM patients is approximately one year following diagnosis, despite multidisciplinary treatment including surgery, $\gamma$-irradiation and chemotherapy $(25,26)$. Therefore, new therapeutic targets are desirable, and ASPM represents a new potential molecular target for the treatment of GBMs (21).

Independently of the studies of Horvath et al (21), we screened an extensive panel of 15 LGA and 15 GBM (27) for microcephalin and ASPM expression by semi-quantitative RT-PCR, Western blotting and immunohistochemistry. Whereas microcephalin mRNA expression was not altered in GBM tissue compared to LGA or normal brain (NB), we confirmed that ASPM mRNA was strongly overexpressed in
GBM. In contrast, ASPM protein was only marginally detected in approximately $13 \%$ of GBM biopsies from patients by Western blotting. However, immunohistochemistry revealed that ASPM protein was expressed very heterogenously in the nuclei of $2 / 3$ of the tumor cells. Primary GBM cells in culture increased their ASPM protein expression with increasing passage number, and GBM cell lines displayed strong ASPM expression, even in the cytoplasm. These findings are of importance both for the use of ASPM expression as a marker for glioma malignancy and as a potential therapeutic target.

\section{Materials and methods}

Tissue samples. Expression of microcephalin and ASPM was analysed using both normal brain and astrocytic tumor specimens. Informed consent of the patient was obtained for the acquisition of tumor material as approved by the local ethics committee. Three samples of temporal brain tissue (normal brain, NB), derived from patients with epilepsy, were kindly provided by Thomas Freiman (University Hospital Freiburg im Breisgau, Germany). The 15 low-grade astrocytomas WHO grade II (LGA) and the 15 glioblastoma multiforme WHO grade IV (GBM) were obtained from patients treated in the University Hospital Würzburg. The GBM patients underwent surgical tumor resection followed by radiotherapy and temozolomid chemotherapy. However, two GBM samples (2369 and 2423) were from recurrent tumors pretreated with $\gamma$-irradiation and chemotherapy. Details concerning patient sex, age, diagnosis, location of tumor and treatment regimen are provided in Stojic et al (27).

Immediately after surgery, tissue samples were frozen at $-80^{\circ} \mathrm{C}$ and then stored in liquid nitrogen. The classification of human brain tumors used in this study was determined by routine histology based on criteria of the World Health Organisation (28).

Cells and cell culture. Primary cells from human GBM were prepared as described elsewhere (29). Human GBM cell lines U87, U251 and U373 were purchased from ATCC (American Type Culture Collection, Rockville, MD), and GaMG was established from a patient with glioblastoma multiforme (Gade Institut of the University Bergen, Norway) (30). Cell lines and primary cells were grown under the same conditions as reported elsewhere (31) in $75 \mathrm{~cm}^{2}$ flasks (Corning, NY, USA) at $37^{\circ} \mathrm{C}, 5.0 \% \mathrm{CO}_{2}$ and $100 \%$ humidity. Primary cells were grown to $90 \%$ confluency. Subsequently, cells were trypsinised (0.25\% trypsin-EDTA; Invitrogen, Carlsbad, CA, USA) and split 1:2. At passage P1 (6 days of culture) and P5 (22 days of culture for tumors 2262 and 2487 and 34 days for tumor 2369), half of the cells were lysed as described below for protein extraction.

RNA and protein extraction. Trypsinised cells were washed twice with phosphate-buffered saline (PBS) and then resuspended in $50 \mu \mathrm{l} \mathrm{PBS}$. Total mRNA and protein were purified from these cells and from $30 \mathrm{mg}$ of surgical specimens, respectively, by the Nucleo-Spin RNA/Protein Kit (Macherey-Nagel, Düren, Germany) according to the manufacturer's instructions. During extraction, RNA was 
treated with RNase-free DNase I to eliminate DNA contaminations in the samples, as recommended by the manufacturer. The RNA samples were eluted with $60 \mu 1$ RNase-free water, and proteins were solubilized in $100 \mu 1$ Protein Loading Buffer (PLB) containing $50 \mathrm{mM}$ Tris (2carboxyethyl) phosphine hydrochloride (TCEP) reducing agent. Purified RNA samples were stored at $-80^{\circ} \mathrm{C}$ and protein/PLB-TCEP mixtures at $-20^{\circ} \mathrm{C}$.

Semi-quantitative RT-PCR analysis. Expression of both microcephalin and ASPM mRNA was analysed by semiquantitative RT-PCR. Total mRNA was extracted as described above and transcribed as described previously (27). The amount of cDNA was normalized to the amount of the housekeeping gene $\beta$-actin. cDNA amplification and expression analysis were performed using primers 5'-AAA GAA GCG GTT GGT CTG AA-3' (forward) and 5'-GGC TCC TCA GAA ATC CAG TG-3' (reverse) for microcephalin and 5'-GTC TGA GCC AGC GAA ATA GG-3' (forward) and 5'-ACT CTG GGC CAT GTT CTC AC-3' (reverse) for ASPM, respectively. These primers were designed in flanking exons with Primer3 software (available online at http://frodo.wi. mit.edu/cgi-bin/primer3/primer3_www.cgi). The polymerase chain reaction was performed using $2.5 \mathrm{U}$ Taq polymerase in each $25 \mu 1$ reaction, containing $10 \mathrm{X}$ buffer with $1.5 \mathrm{mM}$ $\mathrm{MgCl}_{2}$ (Eppendorf, Hamburg, Germany). PCR conditions were optimized for each primer pair to ensure that the comparison between samples was conducted in the linear range. Thermocycle parameters were as follows: $5 \mathrm{~min}$ at $94^{\circ} \mathrm{C} ; 32$ cycles of $30 \mathrm{sec}$ at $94^{\circ} \mathrm{C}, 30 \mathrm{sec}$ at $57^{\circ} \mathrm{C}$ and $59^{\circ} \mathrm{C}$ for microcephalin and ASPM, respectively, $30 \mathrm{sec}$ at $72^{\circ} \mathrm{C}$; and $10 \mathrm{~min}$ at $72^{\circ} \mathrm{C}$. The amplification products were separated on $1 \%$ agarose gels (Sigma-Aldrich, Steinheim, Germany) containing $0.07 \mu \mathrm{g}$ / $\mathrm{ml}$ ethidium bromide (Roth, Karlsruhe, Germany).

Western blotting. Expression of ASPM at the protein level was determined by Western blotting. In total, 3-10 $\mu 1$ of the protein/ PLB-TCEP mixture per lane was separated on a $3-8 \%$ polyacrylamide-tris-acetate gel (Invitrogen) and transferred to a nitrocellulose membrane (Schleicher \& Schüll, Dassel, Germany). After blocking in 5\% milk powder, the membranes were incubated with rabbit polyclonal anti-ASPM antibody (Bethyl Laboratories Inc., Montgomery, TX, USA), diluted 1:500 in TBST (50 mM Tris Base, $150 \mathrm{mM} \mathrm{NaCl}, 0.1 \%$ (v/v) Tween-20) containing 5\% milk powder. Mouse monoclonal anti- $\gamma$-tubulin primary antibody (Sigma, St. Louis, MO, USA) was used at a dilution of 1:5000 in TBST. After washing in TBST, the membrane was incubated with horseradish peroxidase (HRP)-conjugated secondary antibodies: goat anti-rabbit IgG HRP (Santa Cruz, CA, USA) or sheep antimouse IgG HRP (Amersham Pharmacia Biotech, Braunschweig, Germany), both diluted 1:1000 in TBST. Blots were developed in the darkroom using the ECL immunodetection system (Amersham Pharmacia Biotech).

Immunohistochemistry. GBM sections $(2 \mu \mathrm{m})$ were cut from formalin-fixed, paraffin-embedded tissue blocks, placed on Super Frost Plus-coated glass slides (Langenbrinck, Teningen, Germany) and dried overnight at room temperature. Paraffin sections were dewaxed twice with xylene and rehydrated in a graded series of ethanol and in distilled water. The slides were treated with $0.3 \%$ hydrogen peroxide in methanol for 10 min to block endogenous peroxidase activity and blocked with a solution of human immunoglobulin (Beriglobin; Aventis Behring, Marburg, Germany) in PBS (dilution 1:50) for $15 \mathrm{~min}$ at room temperature to reduce unspecific binding capacity. Glioblastoma sections were then incubated overnight at $4^{\circ} \mathrm{C}$ in the presence of primary polyclonal antibodies against ASPM (Bethyl Laboratories Inc., dilution 1:300). After washing with PBS, the sections were incubated with the HRP-labelled LSAB2 kit (streptavidin-biotin system; Dako, Hamburg, Germany). Peroxidase activity was revealed using diaminobenzidine (DAB; Sigma, Deisenhofen, Germany) as a substrate for $5 \mathrm{~min}$, which resulted in brown staining. The slides were counterstained with haematoxylin and were analysed using a light microscope BX41 (Olympus, NY, USA). Negative control experiments were carried out using pre-immune serum instead of primary antibodies.

Statistical analysis. The intensity of the DNA and protein bands was quantified by densitometry using the BioDoc Analyze software (Biometra, Göttingen, Germany), and the DNA and protein bands were normalized with respect to $\beta$-actin and $\gamma$-tubulin respectively. Statistical analysis was performed using Microsoft Office Excel 2003 (Microsoft Deutschland GmbH, Unterschleissheim, Germany). Boxplots were generated using GraphPad Prism 4 Software (GraphPad Software Inc., San Diego, CA, USA).

\section{Results}

ASPM mRNA expression is increased in high grade gliomas, whereas microcephalin $m R N A$ expression is not altered. The expression of both microcephalin and ASPM mRNA is altered in human malignancies $(12,17,20,21)$. However, the expression of both proteins in human gliomas has not been analysed in detail. Therefore, we screened a panel of 3 normal brain (NB), 15 astrocytoma WHO grade II (low-grade astrocytoma, LGA) and 15 glioblastoma multiforme WHO grade IV (GBM) surgical tissue specimens for expression of both microcephalin and ASPM mRNA by semi-quantitative RT-PCR. PCR conditions were optimized for each primer pair to analyze DNA amplification in the linear range. Our results indicate that microcephalin mRNA was expressed at very low levels in all tumor tissues analysed including normal brain (NB) (Fig. 1a). A strong band of the correct size was detected in the positive control (Fig. 1a). In contrast, while ASPM mRNA expression was not detected in NB, it was expressed weakly in LGA and showed strong expression in GBM tissue samples (Fig. 1a). Box-plot analysis of the relative expression levels of microcephalin and ASPM mRNA from NB, LGA and GBM confirmed this observation. Microcephalin was expressed at low levels by NB, LGA and GBM (Fig. 1b), while ASPM mRNA levels showed a positive correlation with the grade of tumor (Fig. 1c). In addition, we observed a high diversity in ASPM mRNA expression among different glioblastoma samples (Fig. 1a). Our analysis did not detect any significant influence of gender, treatment regimen, recurrent versus primary tumor or location of the tumor within the brain on ASPM expression levels in our data set. 

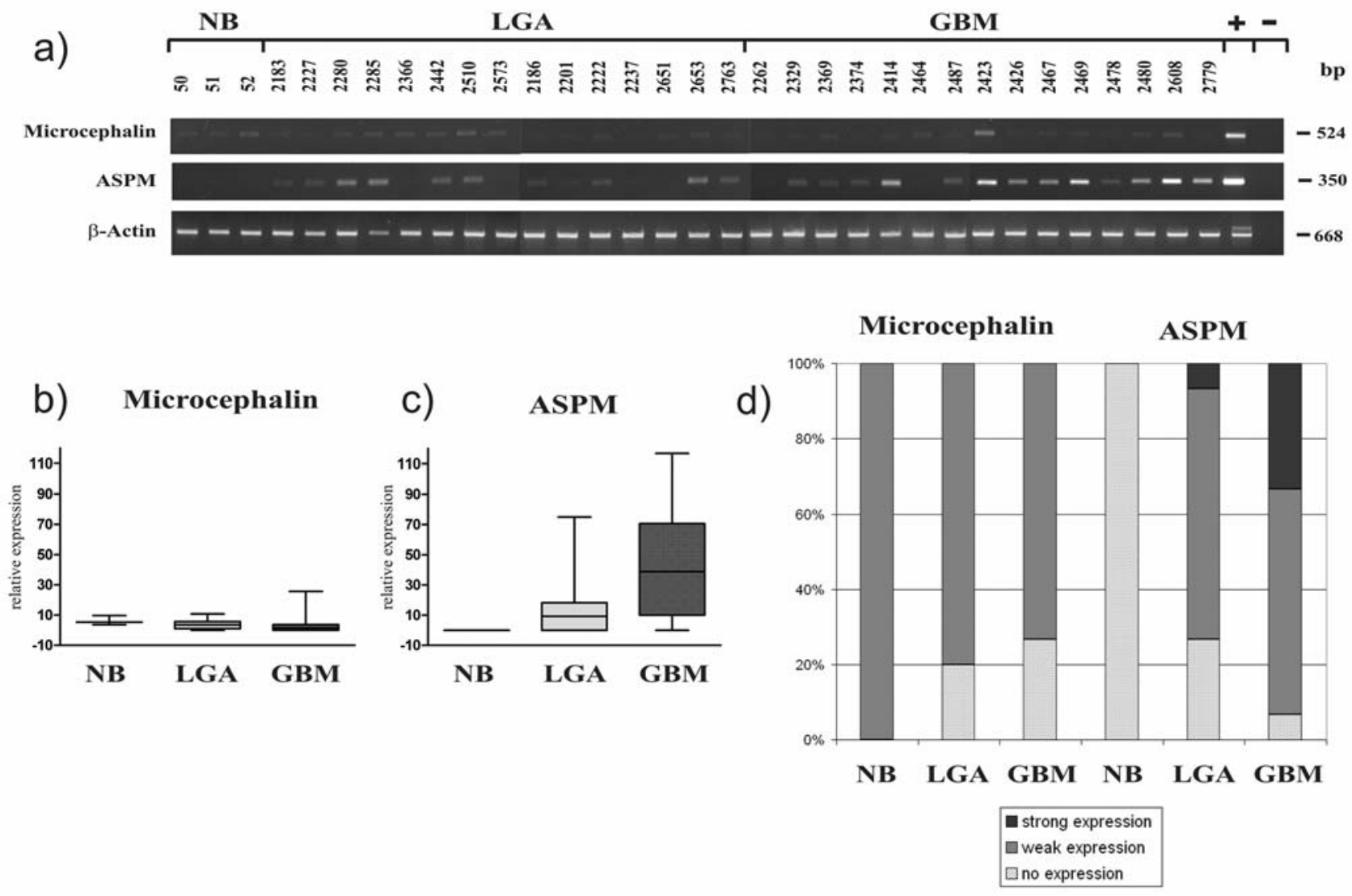

Figure 1. Expression analysis of microcephalin and ASPM in normal brain and human astrocytic tumor samples by semi-quantitative RT-PCR. (a) Total RNA from normal brain (NB), astrocytoma WHO grade II (LGA) and glioblastoma multiforme (GBM) tissue samples was used as a template for RT-PCR analysis. Primers, specific for each transcript, were designed in flanking exons. cDNA from U251 cells was used as the positive control (+). For negative control, cDNA was excluded from the PCR reaction (-). The various cDNA concentrations were normalized to that of the housekeeping gene $B$-actin, which was used as the internal loading control. The size (bp) of the microcephalin, ASPM and B-actin cDNA is indicated at the right. (b) Box-plot analysis of densitometrically quantified microcephalin mRNA expression. Each value was normalized to the respective $\beta$-actin mRNA. The black line within the boxes represents the median expression, boxes show the quartiles and bars indicate minimum and maximum values. (c) Box-plot analysis of densitometrically quantified ASPM mRNA expression. (d) Percentage of tissue samples expressing microcephalin and ASPM mRNA. White dotted columns represent samples with no detectable amounts of mRNA, light grey shows weak expression $[>0$ but $<50$ on a scale from 0 (no expression detectable)] to 100 (strongest expression of ASPM, tumor 2608) and dark grey represents strong expression ( $\geq 50)$.

Kaplan-Meier analysis of strong ASPM mRNA expression versus weak expression did not reveal any significant influence on patient survival rates (data not shown).

However, not only did the level of expression of ASPM mRNA increase with tumor grade, but the number of tumors expressing ASPM mRNA also rose (Fig. 1d). We defined the expression strength on a scale from 0 to 100 (zero meaning no expression detectable, 100 representing the strongest ASPM expression within the panel, which was displayed by tumor 2608). Whereas none of the NB samples expressed ASPM mRNA, $68 \%$ of the LGA samples displayed weak and $7 \%$ strong ASPM mRNA expression (weak expression $>0$ but $<50$ on our scale and strong expression $\geq 50$ ). Of the GBM samples, 7\% did not show any ASPM expression, 60\% displayed weak expression and $33 \%$ displayed strong expression. A similar analysis for microcephalin indicated that there was a small reduction in the number of tumors weakly expressing microcephalin mRNA with increasing tumor grade (NB 100\%, LGA 80\%, GBM 73\%).

In summary, microcephalin mRNA was weakly expressed in all tissue samples analysed, and its level remained unaltered when GBMs were compared with LGA or NB. In contrast, not only did ASPM mRNA levels show a positive correlation with tumor grade, but there was also good correlation between tumor grade and the number of samples with detectable ASPM mRNA.

ASPM protein is strongly expressed by GBM cell lines and in primary cells following extended passage. ASPM protein expression has not been demonstrated in astrocytic tumors. To confirm our RT-PCR data, we analysed the levels of ASPM protein by Western blotting. The same tissue samples that were used for the RT-PCR analysis were also used for immunoblotting. The results of the Western blotting (Fig. 2a) indicated that the ASPM protein was only detectable in 2 of the 15 GBMs (13\%). Expression of ASPM was undetectable in NB, LGA and the majority of the GBM samples (Fig. 2a). Both tissue samples in which ASPM protein was detected were derived from male patients. Patient 2478 survived 29 weeks and patient 2608 survived 47 weeks after diagnosis. However, tissue samples derived from other patients with either shorter or longer survival times did not express the ASPM protein.

ASPM mRNA is overexpressed in human glioblastoma cell lines U251 (17) and U87. siRNA-mediated depletion of 
a)

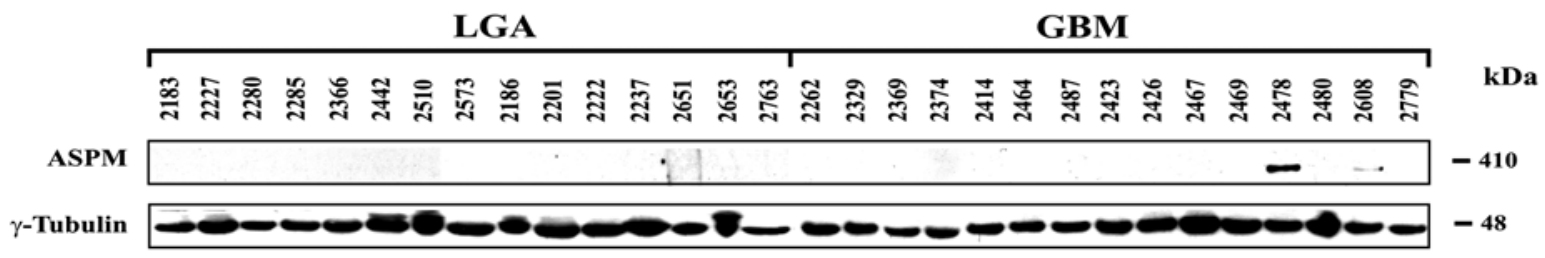

b)

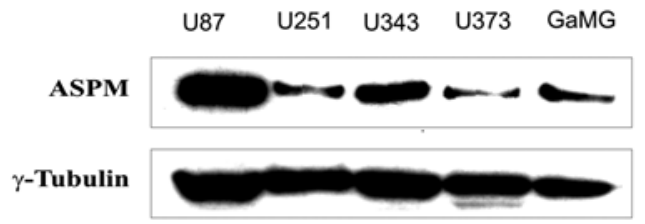

c)

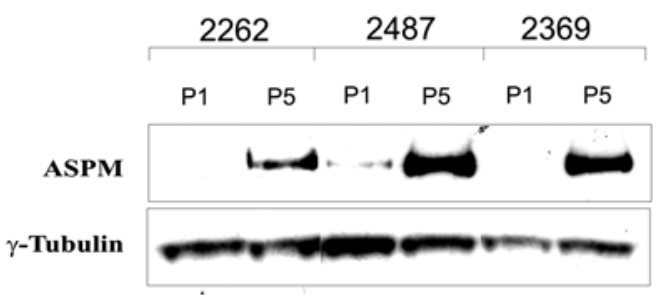

Figure 2. Western blot analysis of ASPM protein expression by human astrocytic tumor samples, human glioblastoma cell lines and primary glioblastoma cells. (a) Protein lysates were isolated from LGA and GBM samples and separated by polyacrylamide gel electrophoresis. Expressed ASPM protein was visualized using specific antibodies. The numbers refer to the tumor samples used (27). (b) ASPM protein expression by the human GBM cell lines U87, U251, U343, U373 and GaMG. (c) Expression of ASPM protein increased with a rise in passages (passage 1 vs. passage 5) of primary GBM cell cultures from tumors 2262, 2369 and 2487. $\gamma$-tubulin served as the loading control in all immunoblots.

ASPM in U87 cells has been shown to inhibit cell proliferation by causing a cell cycle arrest in G1 (21). Therefore, we analysed the expression of ASPM protein in the human GBM cell lines U87, U251, U343, U373 and GaMG by Western blotting (Fig. 2b). All the cell lines tested expressed the ASPM protein at high levels, with U87 cells displaying the strongest expression, followed by U343, GaMG, U251 and U373. The latter three cell lines expressed comparable amounts of ASPM protein. We next analysed primary cell cultures from tumors 2262, 2487 and 2369 for ASPM protein expression by Western blotting both at early passage (P1) and after extended culture (P5). ASPM protein expression was not detected in tumor tissue from which the primary cells were derived (Fig. 2a). Primary cells at P1 displayed either weak or undetectable levels of ASPM protein. However, by P5 the expression of ASPM protein was greatly upregulated (Fig. 2c).

ASPM is expressed in the nuclei of tumor cells in paraffinembedded GBM sections. Although we were unable to detect any ASPM protein expression by Western blotting in the majority of the LGA or GBM tumor samples analysed, we explored the possibility that levels of the ASPM protein may not be present at levels sufficient for detection on an immunoblot. Therefore, we performed immunohistochemistry using paraffin-embedded tissue sections derived from a number of different GBMs. Tissue incubated with pre-immune serum served as the negative control (Fig. 3a). The results of our immunohistochemical analysis suggest that the ASPM protein is expressed heterogenously throughout the tumor section with staining being observed primarily in the nuclei of $\sim 2 / 3$ of the tumor cells (Fig. $3 b$ and c). In the positive control, U87 cells displayed strong ASPM expression primarily in the nucleus but staining was also observed in the cytoplasm (Fig. 3d). In the GBM tissue sections, $\sim 1 / 3$ of all tumor cells, normal cells in the invasion zone, endothelial cells and macrophages were not stained (Fig. 3b). In addition, no correlation was observed between ASPM expression and regions of the tumor such as blood vessels, areas of tissue necrosis, the invasion zone or mitotic cells.

\section{Discussion}

Microcephalin and ASPM are two causative genes for autosomal recessive primary microcephaly (MCPH). Since this disease is characterized by a marked reduction in brain size to approximately the volume found in early hominids (32), both proteins are thought to be involved in the evolution of mammalian brains (33).

Microcephalin is an 835-amino acid protein with a BRCA1 C-terminal (BRCT) domain at the $\mathrm{N}$-terminus and two additional BRCT domains at the C-terminal end of the protein (4). BRCT domains are usually found in factors involved in DNA repair and cell cycle control and participate in both protein-protein and protein-DNA interactions (34). Indeed, a role for microcephalin in the DNA damage response was confirmed by the demonstration that it localizes to DNA repair foci and that cells depleted of microcephalin by RNA interference exhibit a failure of cell cycle checkpoints and show increased genomic instability (9-12). In advanced epithelial ovarian cancer, the genomic instability correlates with loss of the microcephalin gene copy number (12). Approximately $40 \%$ of ovarian cancers have a substantial decrease in microcephalin DNA copy number, and in $63 \%$ of cases microcephalin mRNA levels are reduced (12). A significant decrease in microcephalin mRNA expression has also been observed in a number of breast cancer cell lines and in prostate cancer specimens (12). These data suggest that microcephalin may be a novel tumor suppressor gene. Similar data for astrocytic tumors are not yet available, although such tumors are already highly aneuploid in their 

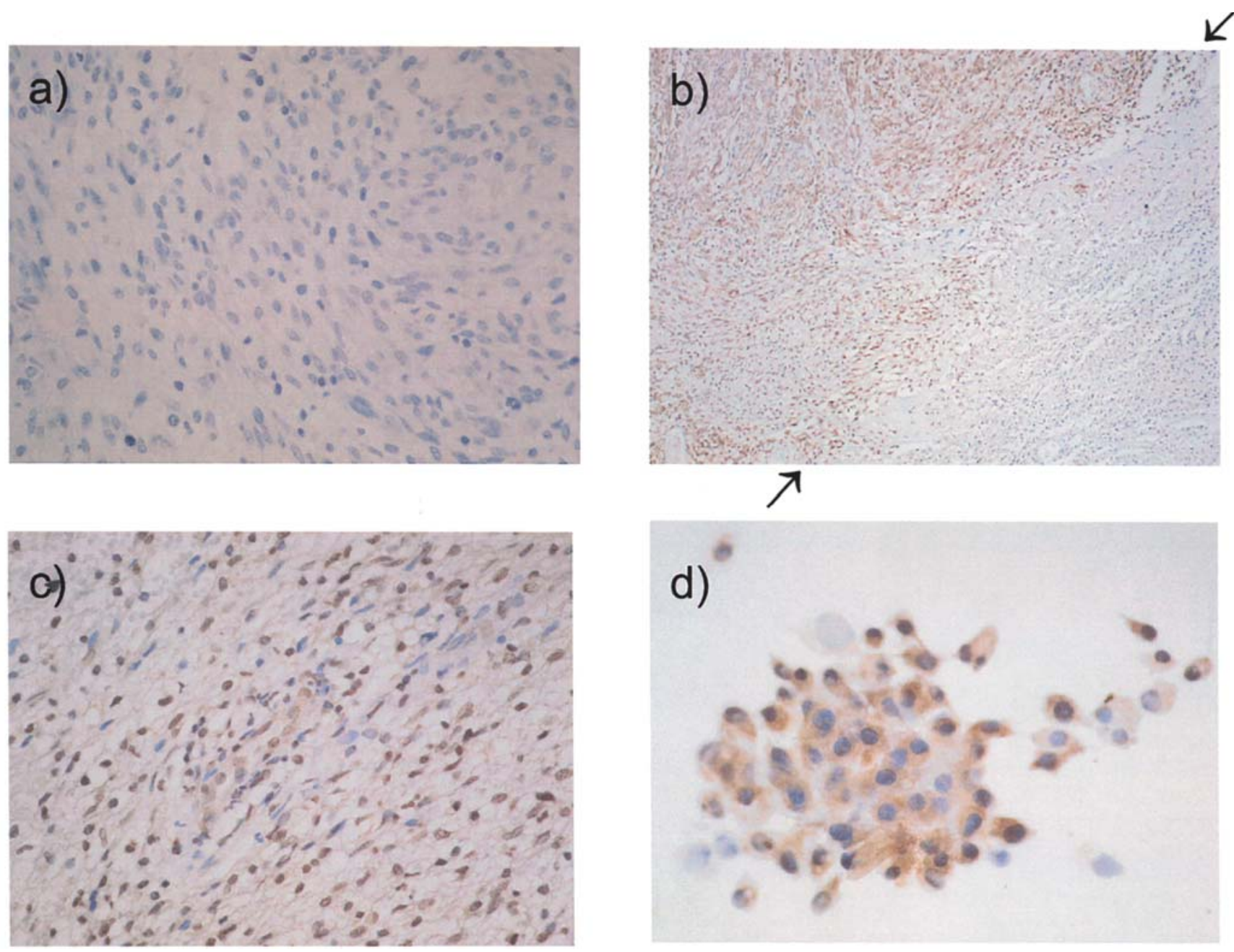

Figure 3. ASPM expression on paraffin-embedded sections of a representative glioblastoma sample and in U87 cells. (a) Tissue incubated with pre-immune serum served as the negative control (magnification, $\mathrm{x} 40$ ). (b, c and d) ASPM expression (brown signal) was visualized by staining with specific antibodies. (b) Heterogenous expression of ASPM by tumor cells throughout the section, but no staining of normal cells within the invasion zone (lower right corner, arrows indicate the invasive front; magnification, $x 10$ ). (c) ASPM was specifically found in the nucleus of $2 / 3$ of the tumor cells (magnification, $x 40$ ). (d) ASPM expression in the U87 glioblastoma cell line was strongly increased and was also found in the cytoplasm of the cells (magnification, $\mathrm{x} 60$ ).

early stages (35-37). To address this lack of data, we screened 15 LGA and 15 GBM by semi-quantitative RT-PCR for microcephalin expression (Fig. 1a). Recently, we showed that this methodology is sufficient to detect alterations in mRNA expression when astrocytic tumors of different WHO grade are compared with each other $(27,38)$. In the current study we found that the number of tumors without detectable microcephalin mRNA increased concomitantly with rising WHO grading (Fig. 1d). Whereas $100 \%$ of NB samples displayed microcephalin mRNA, its expression was reduced to 80 and $73 \%$ in LGA and GBM respectively (Fig. 1d). These percentages are smaller than those detected by Rai et al (12) for ovarian cancers. When the average relative expression levels of microcephalin mRNA in NB, LGA and GBM are compared, then no significant difference is observed (Fig. 1b). Therefore, our data suggest that microcephalin does not play a major role in the development of human gliomas and furthermore that it does not function as a tumor suppressor in this type of cancer.

In contrast, there may be a role for ASPM in the increased aggressiveness of GBMs in comparison to LGA. ASPM is a large protein of 3477 amino acids. It comprises a putative microtubule-binding domain at its $\mathrm{N}$-terminus, a calponin- homology domain, multiple isoleucine-glutamine (IQ) calmodulin-binding domains and a conserved C-terminal region with unknown function $(16,20)$. During embryonic development in mice, ASPM is expressed in the cerebral cortical ventricular zone, the proliferative region of the medial and lateral ganglionic eminence and the ventricular zone of the dorsal diencephalon (16). Expression of ASPM is greatly reduced by the day of birth, when neurogenesis in the cortical ventricular zone is completed and gangliogenesis is increasing, suggesting that ASPM is preferentially expressed in progenitors that produce neurons (16). In these cells, ASPM concentrates at the spindle poles from prophase through telophase (18) and ensures symmetric proliferative divisions of embryonic neuroepithelial cells (19). A reduction in the expression of ASPM inhibits the proliferation of neuronal progenitor cells, thereby reducing the number of neurons formed and resulting in a small brain that is characteristic of MCPH (19). However, in humans, ASPM mRNA is also widely expressed by adult organs such as the breast, lung, pancreas, uterus, colon, thyroid, liver, bladder, kidney, ovary, testis, stomach, lymph node, cervix and esophagus. Only in the adult brain and skeletal muscle there is no detectable expression of ASPM $(17,20)$. ASPM mRNA expression is 
greatly increased in transformed cells and in cancers of the uterus, ovary, breast, colon, thyroid, testis, lymph node and stomach (17). Our semi-quantitative RT-PCR screen of NB, LGA and GBM revealed that there is a clear increase in both the level of ASPM mRNA expression (Fig. 1a and c) and the number of tumors expressing ASPM mRNA (Fig. 1d) that correlates with the WHO grade of the astrocytic tumors. Concurrent with our study, other groups independently published the results of microarray analyses $(22,23)$ and a weighted gene coexpression network analysis (21). They confirmed our findings by showing that ASPM is a key gene within a gene co-expression module that is strongly overexpressed at the mRNA level when compared with either normal brain or control body tissue (21). In addition, the study by Horvath et al demonstrated that siRNA-mediated depletion of ASPM in both the U87 GBM cell line and in low-passage primary GBM cells inhibits proliferation of the cells (21). Therefore, the authors suggested that ASPM may represent a potential molecular therapeutic target. Our own KaplanMeier analysis of strong ASPM mRNA expression versus weak expression did not reveal any significant influence on patient survival rates (data not shown). However, a more detailed survival study would require a larger sample size.

To date, ASPM expression has not been evaluated at the protein level in astrocytic tumors. Therefore, we performed a Western blot analysis on the same panel of human tissues that was used for the determination of mRNA expression (Fig. 2a). Recently, specific antibodies to the ASPM protein have been developed (17). These polyclonal antibodies detect at least four different splice variants of ASPM at $410 \mathrm{kDa}$, $218 \mathrm{kDa}$ and two additional proteins between 150 and $110 \mathrm{kDa}$ in human HT1080 and in HEK293T cells $(17,18)$. The 410- kDa protein represents full-length ASPM $(17,18)$. In our Western blots the 410-kDa isoform of ASPM was clearly observed (Fig. 2), but the other splice variants were not detected (data not shown). The full-length isoform is thought to be brain specific (17). Surprisingly, only two of the 15 GBM tissue samples (13\%) expressed detectable ASPM protein (Fig. 2a). Zhong et al (18) reported that immunohistochemical staining of human breast tumor samples and colorectal carcinoma samples did not reveal any abnormal expression of ASPM when compared to matched tissue controls. In addition, these authors demonstrated that breast cancer and colorectal carcinoma cell lines had lower levels of ASPM protein in comparison with normal epithelial cells (18). These data are in contrast to the clear increase in ASPM mRNA observed in transformed cell lines and tumor tissues $(17,20-23)$ and suggest that ASPM may be regulated at the translational level. However, the results of RNA interference studies suggest that ASPM is involved in the proliferation of GBM cells (21). Since GBM cell lines have not been analysed for ASPM protein expression to date, we analysed total cell lysates from U87, U251, U343, U373 and GaMG by Western blotting (Fig. 2b). U87 cells expressed high levels of ASPM protein, followed by U343, GaMG, U251 and U373 cells (Fig. 2b). Furthermore, our data show that the ASPM protein is also expressed in primary cells derived from GBM patients (Fig. 2c) and is upregulated following extended culture in vitro (Fig. 2c). Our data suggest that ASPM protein expression may correlate with the proliferation state of the cells, and indeed recent immunohistochemical analysis has shown that expression of the ASPM protein is only detected in actively proliferating cells in malignant tissue (17).

Since ASPM has been shown to be a nuclear protein in HT1080, U2OS, HeLa and HaCaT cells $(17,18)$, we performed immunohistochemistry to detect ASPM in paraffin-embedded tissue sections derived from GBM. In 66\% of all tumor cells, clear nuclear staining of ASPM was observed (Fig. $3 b$ and c). ASPM staining was heterogenously distributed throughout the tumor with some areas showing strong staining while others showed only weak ASPM staining. ASPM staining was not associated with any specific areas of the tumor such as blood vessels, invasion zone or mitotic cells. Normal cells in the invasion zone, endothelial cells, macrophages and $33 \%$ of all tumor cells did not express detectable amounts of ASPM (Fig. 3b). That ASPM was expressed in the nuclei of tumor cells may explain why ASPM was not detected in total cell lysate by Western blotting. In cultured cells, on the other hand, ASPM expression was markedly increased, and ASPM was also found strongly expressed in the cytoplasm (Fig. 3d), thereby enabling detection by Western blotting and suggesting that its expression is linked to high cell proliferation rates. In addition, these immunohistochemical data confirm that ASPM is not only overexpressed at the mRNA level, but also at the protein level in the tumors of patients with glioblastoma multiforme.

In summary, whereas microcephalin mRNA expression does not seem to be altered during development of human astrocytic tumors, ASPM is clearly overexpressed at both the mRNA and at the protein level. The overexpression of ASPM correlates with the WHO grade of the gliomas. Therefore, ASPM, which has been shown by others to be related to cell proliferation $(19,21)$, may represent a promising new target for the therapy of these highly aggressive malignancies.

\section{Acknowledgements}

We thank Thomas Freiman (Neurochirurgische Klinik, Neurozentrum, University-Hospital Freiburg im Breisgau, Germany) for the normal brain control tissue. We are grateful to Michaela Kapp (University of Würzburg, Department of Obstetrics and Gynaecology, Würzburg, Germany) for the technical assistance. This project was supported by Interdisziplinäres Zentrum für Klinische Forschung der Universität Würzburg (C.H. and G.H.V.).

\section{References}

1. Mochida GH and Walsh CA: Molecular genetics of human microcephaly. Curr Opin Neurol 14: 151-156, 2001.

2. Woods CG, Bond $\mathrm{J}$ and Enard W: Autosomal recessive primary microcephaly $(\mathrm{MCPH})$ : a review of clinical, molecular, and evolutionary findings. Am J Hum Genet 76: 717-728, 2005.

3. Jackson AP, McHale DP, Campbell DA, Jafri H, Rashid Y, Mannan J, Karbani G, Corry P, Levene MI, Mueller RF, Markham AF, Lench NJ and Woods CG: Primary autosomal recessive microcephaly (MCPH1) maps to chromosome 8p22pter. Am J Hum Genet 63: 541-546, 1998

4. Jackson AP, Eastwood H, Bell SM, Adu J, Toomes C, Carr IA, Roberts E, Hampshire DJ, Crow YJ, Mighell AJ, Karbani G, Jafri H, Rashid Y, Mueller RF, Markham AF and Woods CG: Identification of microcephalin, a protein implicated in determining the size of the human brain. Am J Hum Genet 71: 136-142, 2002. 
5. Lin S-Y and Elledge SJ: Multiple tumor suppressor pathways negatively regulate telomerase. Cell 113: 881-889, 2003.

6. Zhong X, Pfeifer GP and Xu X: Microcephalin encodes a centrosomal protein. Cell Cycle 5: 457-458, 2006.

7. Trimborn M, Bell SM, Felix C, Rashid Y, Jafri H, Griffiths PD, Neumann LM, Krebs A, Reis A, Sperling K, Neitzel H and Jackson AP: Mutations in microcephalin cause aberrant regulation of chromosome cendensation. Am J Hum Genet 75: 261-266, 2004.

8. Trimborn M, Schindler D, Neitzel H and Hirano T: Misregulated chromosome condensation in MCPH1 primary microcephaly is mediated by condensin II. Cell Cycle 5: 322-326, 2006.

9. Xu X, Lee J and Stern DF: Microcephalin is a DNA damage response protein involved in regulation of CHK1 and BRCA1. J Biol Chem 279: 34091-34094, 2004.

10. Lin S-Y, Rai R, Li K, Xu Z-X and Elledge SJ: BRIT1/MCPH1 is a DNA damage responsive protein that regulates the Brca1Chk1 pathway, implicating checkpoint dysfunction in microcephaly. Proc Natl Acad Sci USA 102: 15105-15109, 2005.

11. Alderton GK, Galbiati L, Griffith E, Surinya KH, Neitzel H, Jackson AP, Jeggo PA and O'Driscoll M: Regulation of mitotic entry by microcephalin and its overlap with ATR signalling. Nat Cell Biol 8: 725-733, 2006.

12. Rai R, Dai H, Multani AS, Li K, Chin K, Gray J, Lahad JP, Liang J, Mills GB, Meric-Bernstam F and Lin S-Y: BRIT1 regulates early DNA damage response, chromosomal integrity, and cancer. Cancer Cell 10: 145-157, 2006.

13. Jamieson CR, Fryns J-P, Jacobs J, Matthijs G and Abramowicz MJ: Primary autosomal recessive microcephaly: MCPH5 maps to 1q25-q32. Am J Hum Genet 67: 1575-1577, 2000.

14. Pattison L, Crow YJ, Deeble VJ, Jackson AP, Jafri H, Rashid Y, Roberts E and Woods CG: A fifth locus for primary autosomal recessive microcephaly maps to chromosome 1q31. Am J Hum Genet 67: 1578-1580, 2000 .

15. Cox J, Jackson AP, Bond J and Woods CG: What primary microcephaly can tell us about brain growth. Trends Mol Med 12: 358-366, 2006

16. Bond J, Roberts E, Mochida GH, Hampshire DJ, Scott S, Askham JM, Springell K, Mahadevan M, Crow YJ, Markham AF, Walsh CA and Woods CG: ASPM is a major determinant of cerebral cortical size. Nat Genet 32: 316-320, 2002.

17. Kouprina N, Pavlicek A, Collins NK, Nakano M, Noskov VN, Ohzeki J-I, Mochida GH, Risinger JI, Goldsmith P, Gunsior M, Solomon G, Gersch W, Kim J-H, Barrett JC, Walsh CA, Jurka J, Masumoto $\mathrm{H}$ and Larionov V: The microcephaly ASPM gene is expressed in proliferating tissues and encodes for a mitotic spindle protein. Hum Mol Genet 14: 2155-2165, 2005.

18. Zhong X, Liu L, Zhao A, Pfeifer GP and Xu X: The abnormal spindle-like, microcephaly-associated (ASPM) gene encodes a centrosomal protein. Cell Cycle 4: 1227-1229, 2005.

19. Fish JL, Kosodo Y, Enard W, Pääbo S and Huttner WB: ASPM specifically maintains symmetric proliferative divisions of neuroepithelial cells. Proc Natl Acad Sci USA 103: 10438-10443, 2006.

20. Rhoads A and Kenguele H: Expression of IQ-motif genes in human cells and ASPM domain structure. Ethn Dis 15 (Suppl 5): 88-91, 2005

21. Horvath S, Zhang B, Carlson M, Lu KV, Zhu S, Felciano RM, Laurance MF, Zhao W, Qi S, Chen Z, Lee Y, Scheck AC, Liau LM, Wu H, Geschwind DH, Febbo PG, Kornblum HI, Cloughesy TF, Nelson SF and Mischel PS: Analysis of oncogenic signaling networks in glioblastoma identifies ASPM as a molecular target. Proc Natl Acad Sci USA 103: 17402-17407, 2006.

22. Tso C-L, Freije WA, Day A, Chen Z, Merriman B, Perlina A, Lee Y, Dia EQ, Yoshimoto K, Mischel PS, Liau LM, Cloughesy TF and Nelson SF: Distinct transcription profiles of primary and secondary glioblastoma subgroups. Cancer Res 66: 159-167, 2006.
23. Marie SKN, Okamoto OK, Uno M, Hasegawa APG, Oba-Shinjo SM, Cohen T, Camargo AA, Kosoy A, Carlotti CG Jr, Toledo S, Moreira-Filho CA, Zago MA, Simpson AJ and Caballero OL: Maternal embryonic leucine zipper kinase transcript abundance correlates with malignancy grade in human astrocytomas. Int J Cancer 122: 807-815, 2008.

24. Biernat W, Tohma Y, Yonekawa Y, Kleihues P and Ohgaki H: Alterations of cell cycle regulatory genes in primary (de novo) and secondary glioblastomas. Acta Neuropathol 94: 303-309, 1997.

25. Ohgaki H, Dessen P, Jourde B, Horstmann S, Nishikawa T, Di Patre PL, Burkhard C, Schuler D, Probst-Hensch NM, Maiorka PC, Baeza N, Pisani P, Yonekawa Y, Yasargil MG, Lutolf UM and Kleihues P: Genetic pathways to glioblastoma: a population-based study. Cancer Res 64: 6892-6899, 2004.

26. Stupp R, Mason WP, van den Bent MJ, Weller M, Fisher B, Taphoorn MJ, Belanger K, Brandes AA, Marosi C, Bogdahn U, Curschmann J, Janzer RC, Ludwin SK, Gorlia T, Allgeier A, Lacombe D, Cairncross JG, Eisenhauer E, Mirimanoff RO, European Organisation for Research and Treatment of Cancer Brain Tumor and Radiotherapy Groups, National Cancer Institute of Canada Clinical Trials Group: Radiotherapy plus concomitant and adjuvant temozolomide for glioblastoma. $\mathrm{N}$ Engl J Med 352: 987-996, 2005

27. Stojic J, Hagemann C, Haas S, Herbold C, Kühnel S, Gerngras S, Roggendorf W, Roosen K and Vince GH: Expression of matrix metalloproteinases MMP-1, MMP-11 and MMP-19 is correlated with the WHO-grading of human malignant gliomas. Neurosci Res 60: 40-49, 2008.

28. Kleihues P, Louis DN, Scheithauer BW, Rorke LB, Reifenberger G, Burger PC and Cavenee WK: The WHO classification of tumors of the nervous system. J Neuropathol Exp Neurol 61: 215-225, 2002.

29. Wagner S, Czub S, Greif M, Vince GH, Süss N, Kerkau S, Rieckmann P, Roggendorf W, Roosen K and Tonn JC: Microglial/macrophage expression of interleukin 10 in human glioblastomas. Int J Cancer 82: 12-16, 1999.

30. Akslen LA, Andersen KJ and Bjerkvig R: Characteristics of human and rat glioma cells grown in a defined medium. Anticancer Res 8: 797-803, 1988.

31. Hagemann C, Meyer C, Stojic J, Eicker S, Gerngras S, Kühnel S, Roosen $\mathrm{K}$ and Vince GH: High efficiency transfection of glioma cell lines and primary cells for overexpression and RNAi experiments. J Neurosci Methods 156: 194-202, 2006.

32. Wood B and Collard M: The human genus. Science 284: 65-71, 1999.

33. Ponting $\mathrm{C}$ and Jackson AP: Evolution of primary microcephaly genes and the enlargement of primate brains. Curr Opin Genet Dev 15: 241-248, 2005

34. Huyton T, Bates PA, Zhang X, Sternberg MJ and Freemont PS The BRCA1 C-terminal domain: structure and function. Mutat Res 460: 319-332, 2000.

35. Cianciulli AM, Morace E, Coletta AM, Occhipinti E, Gandolfo GM, Leorardo $\mathrm{G}$ and Carapella CM: Investigation of genetic alterations associated with development and adverse outcome in patients with astrocytic tumor. J Neurooncol 48: 95-101, 2000.

36. Loeper S, Romeike BFM, Heckmann N, Jung V, Henn W, Feiden W, Zhang KD and Urbschat S: Frequent mitotic errors in tumor cells of genetically micro-heterogeneous glioblastomas. Cytogenet Cell Genet 94: 1-8, 2001.

37. Shapiro JR: Genetic alterations associated with adult diffuse astrocytic tumors. Am J Med Genet 115: 194-201, 2002.

38. Said HM, Hagemann C, Staab A, Stojic J, Kühnel S, Vince GH, Flentje M, Roosen K and Vordermark D: Expression patterns of the hypoxia-related genes osteopontin, CA9, erythropoietin, VEGF and HIF- $1 \alpha$ in human glioma in vitro and in vivo. Radiother Oncol 83: 398-405, 2007. 